\title{
Association of genetic variants with hemorrhagic stroke in Japanese individuals
}

\author{
TETSURO YOSHIDA ${ }^{1}$, KIMIHIKO KATO ${ }^{2}$, KIYOSHI YOKOI ${ }^{2}$, MITSUTOSHI OGURI ${ }^{3}$, SACHIRO WATANABE $^{4}$, \\ NORIFUMI METOKI ${ }^{5}$, HIDEMI YOSHIDA ${ }^{6}$, KEI SATOH ${ }^{6}$, YUKITOSHI AOYAGI ${ }^{7}$, \\ YOSHINORI NOZAWA ${ }^{8}$ and YOSHIJI YAMADA ${ }^{9}$
}

\begin{abstract}
${ }^{1}$ Department of Cardiovascular Medicine, Inabe General Hospital, Inabe; ${ }^{2}$ Department of Cardiovascular Medicine, Gifu Prefectural Tajimi Hospital, Tajimi; ${ }^{3}$ Department of Cardiology, Japanese Red Cross Nagoya First Hospital, Nagoya; ${ }^{4}$ Department of Cardiology, Gifu Prefectural General Medical Center, Gifu; ${ }^{5}$ Department of Internal Medicine, Hirosaki Stroke Center, Hirosaki; ${ }^{6}$ Department of Vascular Biology, Institute of Brain Science, Hirosaki University Graduate School of Medicine, Hirosaki; ${ }^{7}$ Department of Genomics for Longevity and Health, Tokyo Metropolitan Institute of Gerontology, Tokyo; ${ }^{8}$ Gifu International Institute of Biotechnology and Tokai Gakuin University, Kakamigahara;

${ }^{9}$ Department of Human Functional Genomics, Life Science Research Center, Mie University, Tsu, Japan
\end{abstract}

Received October 29, 2009; Accepted January 8, 2010

DOI: $10.3892 /$ ijmm_00000388

\begin{abstract}
Although genetic epidemiological studies have implicated several genetic variants as risk factors for hemorrhagic stroke, the genetic determinants of this condition remain largely unknown. We examined an association of genetic variants with intracerebral or subarachnoid hemorrhage among Japanese individuals. The study population comprised 4,304 unrelated Japanese individuals, including 377 subjects with intracerebral hemorrhage, 205 subjects with subarachnoid hemorrhage, and 3,722 controls. The 150 polymorphisms examined in the present study were selected by genome-wide association studies of ischemic stroke and myocardial infarction with the use of the GeneChip Human Mapping 500K Array Set. The chi-square test, multivariable logistic regression analysis with adjustment for covariates, as well as a stepwise forward selection procedure revealed that the $\mathrm{C} \rightarrow \mathrm{T}$ polymorphism (rs1324694) of ERLIN1, the $\mathrm{C} \rightarrow \mathrm{T}$ polymorphism (rs12679196) of TRAPPC9, and the $\mathrm{G} \rightarrow \mathrm{T}$ polymorphism (rs16936752) of WNK2 were significantly $(\mathrm{P}<0.05)$ associated with the prevalence of intracerebral hemorrhage, and that the $\mathrm{A} \rightarrow \mathrm{G}$ polymorphism (rs3111754) of ITM $2 C$ and the $\mathrm{A} \rightarrow \mathrm{G}$ polymorphism (rs 10986769) of $M A P K A P 1$ were significantly associated with the prevalence of subarachnoid hemorrhage. Genotypes for ERLIN1, TRAPPC9, and WNK2 may prove informative for assessment of the genetic risk for intracerebral hemorrhage, and those for ITM2C and MAPKAP1 may be beneficial in assessment of the
\end{abstract}

Correspondence to: Professor Yoshiji Yamada, Department of Human Functional Genomics, Life Science Research Center, Mie University, 1577 Kurima-machiya, Tsu, Mie 514-8507, Japan E-mail: yamada@gene.mie-u.ac.jp

Key words: genetics, polymorphism, hemorrhagic stroke, intracerebral hemorrhage, subarachnoid hemorrhage genetic risk for subarachnoid hemorrhage in Japanese individuals.

\section{Introduction}

Stroke is a complex multifactorial disorder that is thought to result from an interaction between a person's genetic background and various environmental factors. Given that stroke is the leading cause of severe disability and the third leading cause of death, after heart disease and cancer, in Western countries and Japan (1), the identification of biomarkers for stroke risk is important both for risk prediction and for intervention to avert future events.

Although genetic association studies have implicated several loci and candidate genes in predisposition to intracerebral hemorrhage $(2,3)$, subarachnoid hemorrhage, or intracranial aneurysm (4-6), the genes that contribute to genetic susceptibility to these conditions remain to be identified definitively. We previously showed that several gene polymorphisms were associated with intracerebral or subarachnoid hemorrhage in Japanese individuals $(7,8)$. To further examine the genetic factors for these conditions, we have performed an association study for 150 polymorphisms of 144 candidate genes and intracerebral or subarachnoid hemorrhage in 4,304 Japanese individuals. The purpose of the present study was to identify genetic variants that confer susceptibility to intracerebral or subarachnoid hemorrhage in Japanese individuals and thereby to assess the genetic risk of these conditions in such individuals.

\section{Materials and methods}

Study population. The study population comprised 4,304 unrelated Japanese individuals who either visited outpatient clinics of or were admitted to one of the participating hospitals (Gifu Prefectural General Medical Center and Gifu Prefectural Tajimi Hospital in Gifu Prefecture and Hirosaki University 
Hospital, Reimeikyo Rehabilitation Hospital, and Hirosaki Stroke Center in Aomori Prefecture, Japan) between October 2002 and March 2008 because of various symptoms or for an annual health checkup, or who were recruited to a population-based prospective cohort study of aging and agerelated diseases in Nakanojo, Gunma Prefecture, Japan.

The 582 stroke patients included 377 subjects $(245$ men, 132 women) with intracerebral hemorrhage and 205 subjects (89 men, 116 women) with subarachnoid hemorrhage. The stroke patients were recruited from individuals who either were admitted to the participating hospitals because of stroke events or visited outpatient clinics regularly. The diagnosis of intracerebral or subarachnoid hemorrhage was based on the occurrence of a new and abrupt focal neurological deficit, with neurological symptoms and signs persisting for $>24 \mathrm{~h}$; it was confirmed by positive findings in computed tomography or magnetic resonance imaging (or both) of the head. The type of stroke was determined according to the Classification of Cerebrovascular Diseases III (9). Individuals with intracranial hemorrhage from cerebrovascular malformations, moyamoya disease, cerebral venous sinus thrombosis, brain tumors, traumatic cerebrovascular diseases, or subdural hematoma were excluded from enrollment in the study.

The 3,722 control subjects (1,696 men, 2,026 women) were recruited from individuals who visited outpatient clinics of the participating hospitals for common diseases, including hypertension, diabetes mellitus, and hypercholesterolemia, or who were community-dwelling individuals recruited to the prospective cohort study. They had no history of ischemic or hemorrhagic stroke or other cerebral diseases; coronary heart disease, peripheral arterial occlusive disease; or other atherosclerotic, thrombotic, embolic, or hemorrhagic disorders.

The study protocol complied with the Declaration of Helsinki and was approved by the committees on the ethics of Human Research of Mie University Graduate School of Medicine, Hirosaki University Graduate School of Medicine, Gifu International Institute of Biotechnology, Tokyo Metropolitan Institute of Gerontology, and participating hospitals. Written informed consent was obtained from each participant.

Selection of polymorphisms. Our aim was to identify genetic variants associated with hemorrhagic stroke in Japanese individuals in a case-control association study. The 150 polymorphisms examined in the present study (data not shown) were selected by genome-wide association studies of ischemic stroke and myocardial infarction (P-value for allele frequency $\left.<1.0 \times 10^{-7}\right)$ with the use of the GeneChip Human Mapping 500K Array Set (Affymetrix, Santa Clara, CA) (10). We have not examined the relation of these polymorphisms to intracerebral or subarachnoid hemorrhage among Japanese individuals in our previous study $(8,9)$.

Genotyping of polymorphisms. Venous blood $(7 \mathrm{ml})$ was collected into tubes containing $50 \mathrm{mmol} / \mathrm{l}$ ethylenediaminetetraacetic acid (disodium salt), and genomic DNA was isolated with a kit (Genomix; Talent, Trieste, Italy). Genotypes of the 150 polymorphisms were determined at G\&G Science (Fukushima, Japan) by a method that combines the polymerase chain reaction and sequence-specific oligonucleotide probes

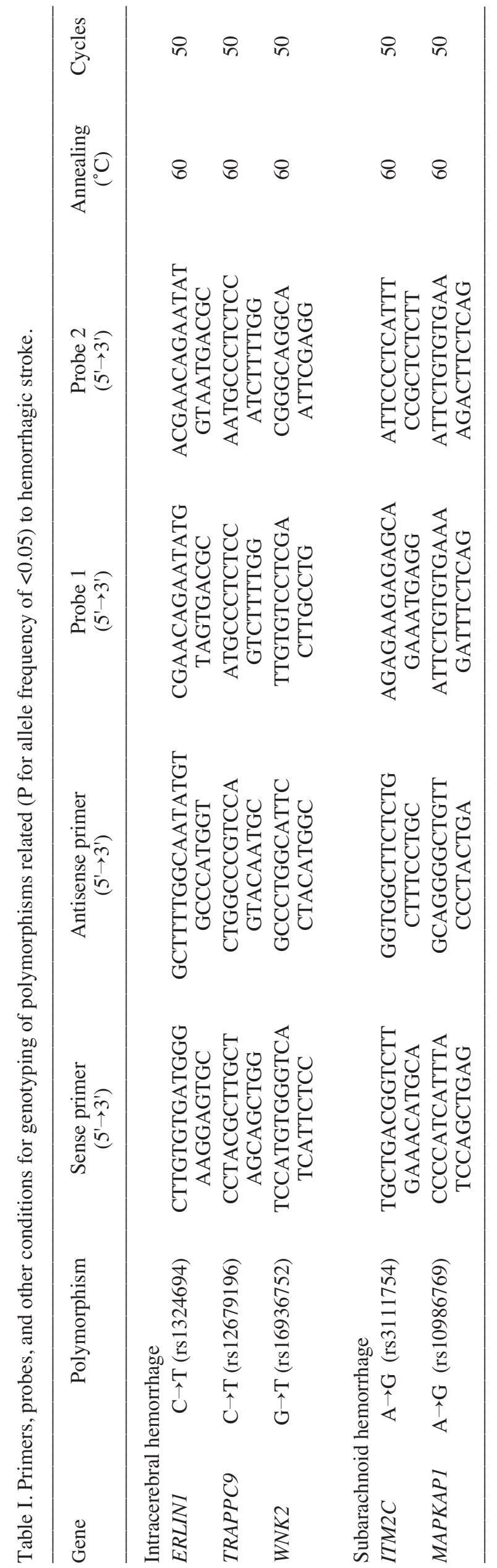


Table II. Characteristics of the 4,304 study subjects.

\begin{tabular}{lccc}
\hline Characteristic & Intracerebral hemorrhage & Subarachnoid hemorrhage & Control \\
\hline No. of subjects & 377 & 205 & 3,722 \\
Age (years) & $64.1 \pm 10.8^{\mathrm{a}}$ & $60.8 \pm 11.9^{\mathrm{a}}$ & $65.8 \pm 11.2$ \\
Sex (male/female, $\%)$ & $65.0 / 35.0^{\mathrm{a}}$ & $43.4 / 56.6$ & $45.6 / 54.4$ \\
Body mass index $\left(\mathrm{kg} / \mathrm{m}^{2}\right)$ & $22.9 \pm 3.8^{\mathrm{b}}$ & $23.0 \pm 3.2$ & $23.4 \pm 3.3$ \\
Current or former smoker $(\%)$ & 20.2 & 19.5 & 19.9 \\
Hypertension $(\%)$ & $72.9^{\mathrm{a}}$ & $56.6^{\mathrm{a}}$ & 41.2 \\
Diabetes mellitus $(\%)$ & $28.1^{\mathrm{a}}$ & $20.0^{\mathrm{b}}$ & 14.3 \\
Hypercholesterolemia $(\%)$ & 20.7 & 21.0 & 24.5 \\
\hline
\end{tabular}

Age and body mass index values are means $\pm \mathrm{SD} .{ }^{\mathrm{a}} \mathrm{P}<0.001,{ }^{\mathrm{b}} \mathrm{P}<0.05$ versus controls.

with suspension array technology (Luminex, Austin, TX). Primers, probes, and other conditions for genotyping of polymorphisms related to intracerebral or subarachnoid hemorrhage are shown in Table I. Detailed genotyping methodology was described previously (11).

Statistical analysis. Quantitative data were compared between subjects with intracerebral or subarachnoid hemorrhage and controls by the unpaired Student's t-test. Categorical data were compared by the chi-square test. Allele frequencies were estimated by the gene counting method, and the chi-square test was used to identify departures from Hardy-Weinberg equilibrium. In the initial screen, the allele frequencies of each polymorphism were compared between subjects with intracerebral or subarachnoid hemorrhage and controls by the chi-square test. Polymorphisms with a P-value for allele frequency of $<0.05$ were further examined by multi-variable logistic regression analysis with adjustment for covariates. Multivariable logistic regression analysis was thus performed with intracerebral or subarachnoid hemorrhage as a dependent variable and independent variables including age, sex $(0$, woman; 1, man), body mass index (BMI), smoking status (0, nonsmoker; 1 , smoker), history of hypertension, diabetes mellitus, and hypercholesterolemia ( 0 , no history; 1 , positive history), and genotype of each polymorphism; and the P-value, odds ratio, and $95 \%$ confidence interval were calculated. Each genotype was assessed according to dominant, recessive, and additive genetic models $(12,13)$. We also performed a stepwise forward selection procedure to examine the effects of genotypes as well as of other covariates on intracerebral or subarachnoid hemorrhage; each genotype was examined according to a dominant or recessive model on the basis of statistical significance in the multivariable logistic regression analysis. A P-value of $<0.05$ was considered statistically significant. Statistical significance was examined by twosided tests performed with JMP version 6.0 and JMP Genomics version 3.2 software (SAS Institute, Cary, NC).

\section{Results}

The characteristics of the 4,304 study subjects are shown in Table II. For individuals with intracerebral hemorrhage, the frequency of male subjects and the prevalence of hypertension and diabetes mellitus were greater, whereas age and BMI were smaller, in subjects with intracerebral hemorrhage than in controls. For individuals with subarachnoid hemorrhage, the prevalence of hypertension and diabetes mellitus were greater, whereas age was younger, in subjects with subarachnoid hemorrhage than in controls.

Comparison of allele frequencies by the chi-square test revealed that the $\mathrm{C} \rightarrow \mathrm{T}$ polymorphism (rs1324694) of ERLINI, $\mathrm{C} \rightarrow \mathrm{G}$ polymorphism (rs199515) of WNT3, $\mathrm{A} \rightarrow \mathrm{C}$ polymorphism (rs9615362) of CELSR1, the $\mathrm{A} \rightarrow \mathrm{C}$ polymorphism (rs942576) of COL13A1, $\mathrm{C} \rightarrow \mathrm{T}$ polymorphism (rs8068452) of CARD14, $\mathrm{A} \rightarrow \mathrm{G}$ polymorphism (rs1671021) of $L L G L 2, \mathrm{~A} \rightarrow \mathrm{C}$ polymorphism (rs4407312) of CST2, $\mathrm{C} \rightarrow \mathrm{T}$ polymorphism (rs12679196) of TRAPPC9, $\mathrm{C} \rightarrow \mathrm{T}$ polymorphism (rs4552453) of SLIT2, and $\mathrm{G} \rightarrow \mathrm{T}$ polymorphism (rs16936752) of WNK2 were significantly $(\mathrm{P}<0.05)$ associated with the prevalence of intracerebral hemorrhage (Table III). Similar analysis revealed the $\mathrm{C} \rightarrow \mathrm{T}$ polymorphism (rs11690358) of $\mathrm{COL} 6 \mathrm{~A} 3, \mathrm{~A} \rightarrow \mathrm{G}$ polymorphism (rs3111754) of ITM2C, $\mathrm{A} \rightarrow \mathrm{G}$ polymorphism (rs10986769) of MAPKAP1, C $\rightarrow \mathrm{T}$ polymorphism (rs4653579) of $N V L, \mathrm{C} \rightarrow \mathrm{G}$ polymorphism (rs199515) of WNT3, and $\mathrm{A} \rightarrow \mathrm{C}$ polymorphism (rs8053843) of TBL3 were significantly associated with the prevalence of subarachnoid hemorrhage (Table III).

Multivariable logistic regression analysis with adjustment for age, sex, BMI, smoking status, and the prevalence of hypertension, diabetes mellitus, and hypercholesterolemia revealed that the $\mathrm{C} \rightarrow \mathrm{T}$ polymorphism (rs1324694) of ERLIN1 (dominant and additive 1 models), $\mathrm{C} \rightarrow \mathrm{T}$ polymorphism (rs12679196) of TRAPPC9 (recessive and additive 2 models), and $\mathrm{G} \rightarrow \mathrm{T}$ polymorphism (rs16936752) of WNK2 (recessive model) were significantly $(\mathrm{P}<0.05)$ associated with intracerebral hemorrhage (Table IV). The $T$ allele of $W N K 2$ was a risk factor for intracerebral hemorrhage, whereas the $T$ alleles of ERLIN1 and TRAPPC9 were protective against this condition. Similar analysis revealed that the $A \rightarrow G$ polymorphism (rs3111754) of ITM2C (recessive model) and $\mathrm{A} \rightarrow \mathrm{G}$ polymorphism (rs10986769) of MAPKAPl (dominant and additive 1 models) were significantly associated with subarachnoid hemorrhage (Table IV). The $G$ allele of MAPKAPI was a risk factor for subarachnoid hemorrhage, whereas the $G$ allele of ITM2C was protective against this condition.

A stepwise forward selection procedure was performed to examine the effects of genotypes for the polymorphisms as 
Table III. Genotype distributions of polymorphisms significantly (P-value for allele frequency <0.05) associated with intracerebral or subarachnoid hemorrhage as determined by the chi-square test.

\begin{tabular}{|c|c|c|c|c|c|}
\hline Gene & Polymorphism & dbSNP & Cases $^{\mathrm{a}}$ & Controls ${ }^{\mathrm{a}}$ & $\mathrm{P}$ (allele frequency) \\
\hline \multicolumn{6}{|c|}{ Intracerebral hemorrhage } \\
\hline \multirow[t]{4}{*}{ ERLIN1 } & $\mathrm{C} \rightarrow \mathrm{T}$ & rs1324694 & & & 0.0024 \\
\hline & $\mathrm{CC}$ & & $326(87.4)$ & $2,983(81.4)$ & \\
\hline & $\mathrm{CT}$ & & $46(12.3)$ & $641(17.5)$ & \\
\hline & $\mathrm{TT}$ & & $1(0.3)$ & $41 \quad(1.1)$ & \\
\hline \multirow[t]{4}{*}{ WNT3 } & $\mathrm{C} \rightarrow \mathrm{G}$ & rs199515 & & & 0.0048 \\
\hline & $\mathrm{CC}$ & & $373(99.5)$ & $3,690(99.9)$ & \\
\hline & $\mathrm{CG}$ & & $2(0.5)$ & $2(0.1)$ & \\
\hline & GG & & $0 \quad(0)$ & $0 \quad(0)$ & \\
\hline \multirow[t]{4}{*}{ CELSRI } & $\mathrm{A} \rightarrow \mathrm{C}$ & rs9615362 & & & 0.0144 \\
\hline & AA & & $1 \quad(0.3)$ & $0(0)$ & \\
\hline & $\mathrm{AC}$ & & $14(3.7)$ & $82 \quad(2.2)$ & \\
\hline & $\mathrm{CC}$ & & $360(96.0)$ & $3,610(97.8)$ & \\
\hline \multirow[t]{4}{*}{ COL13A1 } & $\mathrm{A} \rightarrow \mathrm{C}$ & rs942576 & & & 0.0171 \\
\hline & AA & & $12(3.2)$ & $72(2.0)$ & \\
\hline & $\mathrm{AC}$ & & $98(26.3)$ & $827(22.5)$ & \\
\hline & $\mathrm{CC}$ & & $263(70.5)$ & $2,773(75.5)$ & \\
\hline \multirow[t]{4}{*}{ CARD14 } & $\mathrm{C} \rightarrow \mathrm{T}$ & rs 8068452 & & & 0.0212 \\
\hline & $\mathrm{CC}$ & & 44 (11.7) & $329 \quad(8.9)$ & \\
\hline & $\mathrm{CT}$ & & $161(42.9)$ & $1,496(40.5)$ & \\
\hline & TT & & $170(45.4)$ & $1,867(50.6)$ & \\
\hline \multirow[t]{4}{*}{$L L G L 2$} & $\mathrm{~A} \rightarrow \mathrm{G}$ & rs1671021 & & & 0.0227 \\
\hline & $\mathrm{AA}$ & & $293(77.7)$ & $2,713(73.2)$ & \\
\hline & $\mathrm{AG}$ & & $82(21.8)$ & $919(24.8)$ & \\
\hline & GG & & $2(0.5)$ & $76 \quad(2.0)$ & \\
\hline \multirow[t]{4}{*}{ CST2 } & $\mathrm{A} \rightarrow \mathrm{C}$ & rs4407312 & & & 0.0235 \\
\hline & $\mathrm{AA}$ & & $3(0.8)$ & $37 \quad(1.0)$ & \\
\hline & $\mathrm{AC}$ & & $38(10.2)$ & $533(14.5)$ & \\
\hline & $\mathrm{CC}$ & & $332(89.0)$ & $3,102(84.5)$ & \\
\hline \multirow[t]{4}{*}{ TRAPPC9 } & $\mathrm{C} \rightarrow \mathrm{T}$ & rs12679196 & & & 0.0322 \\
\hline & $\mathrm{CC}$ & & $250(66.5)$ & $2,296(62.5)$ & \\
\hline & $\mathrm{CT}$ & & $120(31.9)$ & $1,218(33.2)$ & \\
\hline & $\mathrm{TT}$ & & $6(1.6)$ & $157 \quad(4.3)$ & \\
\hline \multirow[t]{4}{*}{ SLIT2 } & $\mathrm{C} \rightarrow \mathrm{T}$ & rs4552453 & & & 0.0383 \\
\hline & $\mathrm{CC}$ & & 372 (99.2) & $3,684(99.8)$ & \\
\hline & $\mathrm{CT}$ & & $3(0.8)$ & $8(0.2)$ & \\
\hline & $\mathrm{TT}$ & & $0 \quad(0)$ & $0(0)$ & \\
\hline \multirow[t]{4}{*}{$W N K 2$} & $\mathrm{G} \rightarrow \mathrm{T}$ & rs 16936752 & & & 0.0406 \\
\hline & GG & & 4 (1.1) & $46 \quad(1.2)$ & \\
\hline & GT & & 55 (14.6) & $700(19.1)$ & \\
\hline & $\mathrm{TT}$ & & $317(84.3)$ & $2,925(79.7)$ & \\
\hline \multicolumn{6}{|c|}{ Subarachnoid hemorrhage } \\
\hline \multirow[t]{4}{*}{ COL6A3 } & $\mathrm{C} \rightarrow \mathrm{T}$ & rs11690358 & & & 0.0048 \\
\hline & $\mathrm{CC}$ & & $0 \quad(0)$ & $0 \quad(0)$ & \\
\hline & $\mathrm{CT}$ & & $10 \quad(4.9)$ & $73 \quad(2.0)$ & \\
\hline & $\mathrm{TT}$ & & $193(95.1)$ & $3,619(98.0)$ & \\
\hline \multirow[t]{4}{*}{ ITM2C } & $\mathrm{A} \rightarrow \mathrm{G}$ & rs3111754 & & & 0.0070 \\
\hline & $\mathrm{AA}$ & & $11(5.4)$ & $150 \quad(4.1)$ & \\
\hline & $\mathrm{AG}$ & & $84(41.4)$ & $1,216(32.9)$ & \\
\hline & GG & & $108(53.2)$ & $2,326(63.0)$ & \\
\hline MAPKAP1 & $\mathrm{A} \rightarrow \mathrm{G}$ & rs10986769 & & & 0.0173 \\
\hline
\end{tabular}


Table III. Continued.

\begin{tabular}{|c|c|c|c|c|c|}
\hline Gene & Polymorphism & dbSNP & Cases $^{\mathrm{a}}$ & Controls $^{\mathrm{a}}$ & $\mathrm{P}$ (allele frequency) \\
\hline & AA & & $133(64.9)$ & $2,669(72.7)$ & \\
\hline & $\mathrm{AG}$ & & $66(32.2)$ & 925 (25.2) & \\
\hline & GG & & $6 \quad(2.9)$ & $78 \quad(2.1)$ & \\
\hline \multirow[t]{4}{*}{$N V L$} & $\mathrm{C} \rightarrow \mathrm{T}$ & rs4653579 & & & 0.0194 \\
\hline & $\mathrm{CC}$ & & $166(81.4)$ & $3,202(87.2)$ & \\
\hline & $\mathrm{CT}$ & & $37(18.1)$ & 455 (12.4) & \\
\hline & $\mathrm{TT}$ & & $1 \quad(0.5)$ & $14(0.4)$ & \\
\hline \multirow[t]{4}{*}{ WNT3 } & $\mathrm{C} \rightarrow \mathrm{G}$ & rs199515 & & & 0.0284 \\
\hline & $\mathrm{CC}$ & & $202(99.5)$ & 3,690 (99.9) & \\
\hline & $\mathrm{CG}$ & & $1(0.5)$ & $2(0.1)$ & \\
\hline & GG & & $0(0)$ & $0 \quad(0)$ & \\
\hline \multirow[t]{4}{*}{$T B L 3$} & $\mathrm{~A} \rightarrow \mathrm{C}$ & rs8053843 & & & 0.0331 \\
\hline & $\mathrm{AA}$ & & $185(91.1)$ & $3,178(86.1)$ & \\
\hline & $\mathrm{AC}$ & & 18 (8.9) & 495 (13.4) & \\
\hline & $\mathrm{CC}$ & & $0 \quad(0)$ & $19(0.5)$ & \\
\hline
\end{tabular}

${ }^{\mathrm{a} N u m b e r s ~ i n ~ p a r e n t h e s e s ~ a r e ~ p e r c e n t a g e s . ~}$

well as of age, sex, BMI, smoking status, and the prevalence of hypertension, diabetes mellitus, and hypercholesterolemia on intracerebral or subarachnoid hemorrhage (Table V). Hypertension, diabetes mellitus, male sex, BMI, ERLIN1 genotype (dominant model), TRAPPC9 genotype (recessive model), WNK2 genotype (recessive model), age, and LLGL2 genotype (dominant model), in descending order of statistical significance, were significant $(\mathrm{P}<0.05)$ and independent determinants of intracerebral hemorrhage. Hypertension, age, BMI, female sex, diabetes mellitus, MAPKAP1 genotype (dominant model), and ITM2C genotype (recessive model) were significant and independent determinants of subarachnoid hemorrhage.

Finally, we examined whether the genotype distributions for the polymorphisms associated with hemorrhagic stroke were in Hardy-Weinberg equilibrium. The genotype distributions for controls of five polymorphisms significantly associated with intracerebral or subarachnoid hemorrhage were in Hardy-Weinberg equilibrium (Table VI).

\section{Discussion}

We examined the possible relations of 150 polymorphisms of 144 candidate genes to the prevalence of intracerebral or subarachnoid hemorrhage in Japanese individuals. Our association study with three steps of analysis (chi-square test, multivariable logistic regression analysis with adjustment for covariates, and stepwise forward selection procedure) revealed that the $\mathrm{C} \rightarrow \mathrm{T}$ polymorphism (rs1324694) of ERLIN1, C $\rightarrow$ T polymorphism (rs12679196) of TRAPPC9, and $\mathrm{G} \rightarrow \mathrm{T}$ polymorphism (rs16936752) of WNK2 were significantly associated with intracerebral hemorrhage, and that the $A \rightarrow G$ polymorphism (rs3111754) of ITM2C and $\mathrm{A} \rightarrow \mathrm{G}$ polymorphism (rs10986769) of MAPKAPl were significantly associated with subarachnoid hemorrhage.
ER lipid raft associated 1 (ERLIN1) has an N-terminal mitochondrial targeting sequence, followed by a transmembrane region and sites for phosphorylation, N-myristoylation, N-glycosylation, and glycosaminoglycan attachment (14). ERLIN1 is a component of lipid rafts and localized specifically to the endoplasmic reticulum and nuclear envelope (15). We have now shown that the $\mathrm{C} \rightarrow \mathrm{T}$ polymorphism (rs1324694) in 5' region of ERLIN1 was significantly associated with intracerebral hemorrhage in Japanese individuals, with the $T$ allele protecting against this condition.

Trafficking protein particle complex 9 (TRAPPC9) binds mitogen-activated protein kinase kinase kinase 14 and inhibitor of $\kappa$ light polypeptide gene enhancer in B-cells, kinase $\beta$, and plays a role in a signaling pathway of the neuronal NFKB1 (16). We have now shown that the $\mathrm{C} \rightarrow \mathrm{T}$ polymorphism (rs12679196) in intron 21 of TRAPPC9 was significantly associated with intracerebral hemorrhage in Japanese individuals, with the $T$ allele protecting against this condition.

WNK lysine deficient protein kinase 2 (WNK2) is a cytoplasmic serine-threonine kinase that contains cysteine in place of the lysine found at the conserved ATP-binding location in subdomain II of protein kinases. WNK2 is involved in the modulation of growth factor-induced cancer cell proliferation through the mitogen-activated protein kinase kinase 1/mitogenactivated protein kinase 14 pathway (17). We have now shown that the $\mathrm{G} \rightarrow \mathrm{T}$ polymorphism (rs16936752) in intron 25 of WNK2 was significantly associated with intracerebral hemorrhage in Japanese individuals, with the $T$ allele representing a risk factor for this condition.

Integral membrane protein 2C (ITM2C) consists of integral type II membrane glycoproteins with an extracellular C terminus (18). In situ hybridization analysis showed that ITM2C and B-amyloid protein-converting enzyme were expressed with overlapping patterns in the brains of an Alzheimer disease patient and a mouse model of this disease 


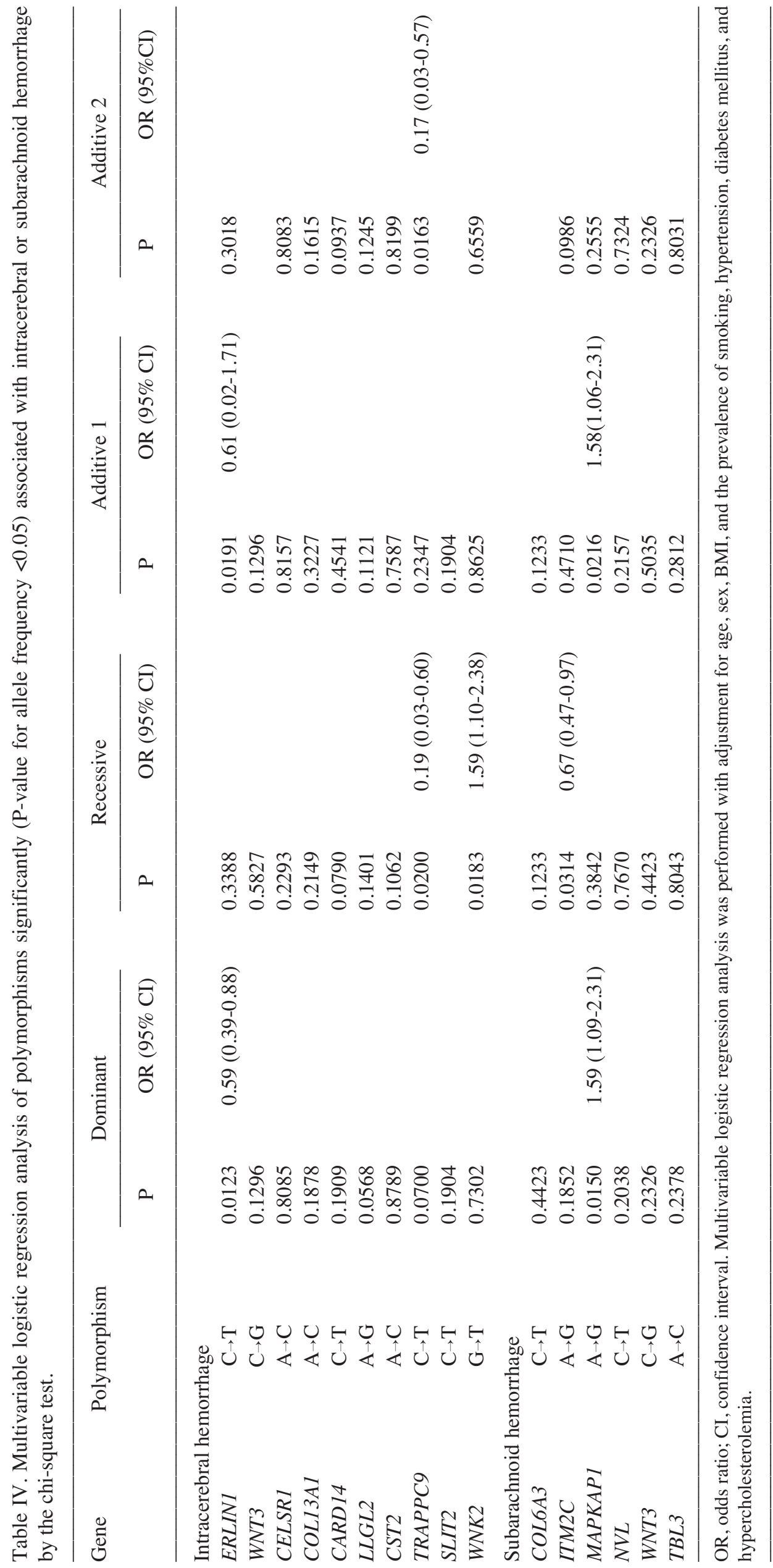


Table V. Effects of genotypes and other characteristics on intracerebral or subarachnoid hemorrhage determined by a stepwise forward selection procedure $(\mathrm{P}<0.05)$.

\begin{tabular}{lcc}
\hline Variable & $\mathrm{P}$ & $\mathrm{R}^{2}$ \\
\hline Intracerebral hemorrhage & & \\
Hypertension & $<0.0001$ & 0.1266 \\
Diabetes mellitus & $<0.0001$ & 0.0195 \\
Male sex & $<0.0001$ & 0.0124 \\
BMI & 0.0001 & 0.0088 \\
ERLIN1 $(C T+T T$ versus $C C)$ & 0.0027 & 0.0052 \\
TRAPPC9 $(T T$ versus $C C+C T)$ & 0.0054 & 0.0045 \\
WNK2 (TT versus $G G+G T)$ & 0.0092 & 0.0039 \\
Age & 0.0114 & 0.0036 \\
LLGL2 $(A G+G G$ versus $A A)$ & 0.0328 & 0.0026 \\
Subarachnoid hemorrhage & & \\
Hypertension & $<0.0001$ & 0.0821 \\
Age & $<0.0001$ & 0.0234 \\
BMI & 0.0010 & 0.0100 \\
Female sex & 0.0129 & 0.0057 \\
Diabetes mellitus & 0.0260 & 0.0046 \\
MAPKAP1 $(A G+G G$ versus $A A)$ & 0.0319 & 0.0042 \\
$I T M 2 C(G G$ versus $A A+A G)$ & 0.0465 & 0.0037 \\
\hline
\end{tabular}

$\mathrm{R}^{2}$, contribution rate.

(19). We have now shown that the $A \rightarrow G$ polymorphism (rs3111754) in intron 1 of ITM2C was significantly associated with subarachnoid hemorrhage in Japanese individuals, with the $G$ allele protecting against this condition.

Mitogen-activated protein kinase-associated protein 1 (MAPKAP1), mechanistic target of rapamycin, RPTOR independent companion of MTOR, complex 2, and MTOR associated protein, LST8 homolog are components of CREB regulated transcription coactivator 2 that is a protein kinase complex involved in phosphorylation and cell signaling through v-akt murine thymoma viral oncogene homolog 1 (20). We have now shown that the $\mathrm{A} \rightarrow \mathrm{G}$ polymorphism (rs10986769) in intron 9 of MAPKAPl was significantly associated with subarachnoid hemorrhage in Japanese individuals, with the $G$ allele representing a risk factor for this condition.

Our study has several limitations: (i) It is possible that one or more of the polymorphisms associated with hemorrhagic stroke in the present study are in linkage disequilibrium with other polymorphisms in the same gene or in other nearby genes that are actually responsible for the development of this condition. (ii) The functional relevance of the identified polymorphisms to gene transcription or to protein structure or function was not determined in the present study. (iii) Given the multiple comparisons of genotypes with hemorrhagic stroke, it is not possible to exclude completely potential statistical errors such as false positives. (iv) The results of the present study were not replicated in independent subject panels.

In conclusion, our present results suggest that ERLIN1, TRAPPC9, and WNK2 may be susceptibility loci for intra-
Table VI. Hardy-Weinberg P-values in subjects with hemorrhagic stroke and controls.

\begin{tabular}{lcccc}
\hline Gene & SNP & dbSNP & Cases & Controls \\
\hline \multicolumn{5}{l}{ Intracerebral hemorrhage } \\
ERLIN1 & $\mathrm{C} \rightarrow \mathrm{T}$ & $\mathrm{rs} 1324694$ & 0.6397 & 0.3208 \\
TRAPPC9 & $\mathrm{C} \rightarrow \mathrm{T}$ & $\mathrm{rs} 12679196$ & 0.0466 & 0.7769 \\
WNK2 & $\mathrm{G} \rightarrow \mathrm{T}$ & $\mathrm{rs} 16936752$ & 0.3605 & 0.5735 \\
Subarachnoid hemorrhage & & \\
ITM2C & $\mathrm{A} \rightarrow \mathrm{G}$ & $\mathrm{rs} 3111754$ & 0.3019 & 0.5705 \\
MAPKAP1 & $\mathrm{A} \rightarrow \mathrm{G}$ & $\mathrm{rs} 10986769$ & 0.5199 & 0.8376 \\
\hline
\end{tabular}

cerebral hemorrhage, and that ITM2C and MAPKAP1 may constitute such loci for subarachnoid hemorrhage in Japanese individuals. Genotypes for these polymorphisms may prove informative for assessment of genetic risk for intracerebral or subarachnoid hemorrhage in such individuals. Given that our present study may be considered as hypothesis generating, validation of our findings will require their replication with independent subject panels.

\section{Acknowledgements}

This work was supported in part by Grants-in-Aid for Scientific Research from the Ministry of Education, Culture, Sports, Science, and Technology of Japan (nos. 18209023, 18018021, and 19659149 to Y. Yamada).

\section{References}

1. Warlow C, Sudlow C, Dennis M, Wardlaw J and Sandercock P: Stroke. Lancet 362: 1211-1224, 2003.

2. O'Donnell HC, Rosand J, Knudsen KA, et al: Apolipoprotein E genotype and the risk of recurrent lobar intracerebral hemorrhage. N Engl J Med 342: 240-245, 2000.

3. Wang Y, Zhang W, Zhang Y, et al: VKORC1 haplotypes are associated with arterial vascular diseases (stroke, coronary heart disease, and aortic dissection). Circulation 113: 1615-1621, 2006.

4. Inoue K, Mineharu Y, Inoue S, et al: Search on chromosome 17 centromere reveals TNFRSF13B as a susceptibility gene for intracranial aneurysm: a preliminary study. Circulation 113: 2002-2010, 2006.

5. Ruigrok YM, Rinkel GJ, van't Slot R, Wolfs $M$, Tang S and Wijmenga C: Evidence in favor of the contribution of genes involved in the maintenance of the extracellular matrix of the arterial wall to the development of intracranial aneurysms. Hum Mol Genet 15: 3361-3368, 2006.

6. Bilguvar K, Yasuno K, Niemelä M, et al: Susceptibility loci for intracranial aneurysm in European and Japanese populations. Nat Genet 40: 1472-1477, 2008.

7. Yamada Y, Metoki N, Yoshida H, et al: Genetic factors for ischemic and hemorrhagic stroke in Japanese individuals. Stroke 39: 2211-2218, 2008.

8. Yamada Y, Metoki N, Yoshida $\mathrm{H}$, et al: Genetic risk for ischemic and hemorrhagic stroke. Arterioscler Thromb Vasc Biol 26: 1920-1925, 2006.

9. A committee established by the Director of the National Institute of Neurological Disorders and Stroke, National Institutes of Health: Special report from the National Institute of Neurological Disorders and Stroke. Classification of Cerebrovascular Diseases III. Stroke 21: 637-676, 1990.

10. Yamada Y, Fuku N, Tanaka M, et al: Identification of CELSRI as a susceptibility gene for ischemic stroke in Japanese individuals by a genome-wide association study. Atherosclerosis 207: 144-149, 2009. 
11. Itoh Y, Mizuki N, Shimada T, et al: High throughput DNA typing of HLA-A, -B, -C and -DRB1 loci by a PCR-SSOP-Luminex method in the Japanese population. Immunogenetics 57: 717-729, 2005.

12. Yamada $\mathrm{Y}$, Kato K, Oguri M, et al: Association of genetic variants with atherothrombotic cerebral infarction among Japanese individuals with metabolic syndrome. Int J Mol Med 21: 801-808, 2008.

13. Oguri M, Kato K, Yokoi K, et al: Association of a polymorphism of $B C H E$ with ischemic stroke in Japanese individuals with chronic kidney disease. Mol Med Rep 2: 779-785, 2009.

14. Browman DT, Resek ME, Zajchowski LD and Robbins SM: Erlin-1 and erlin-2 are novel members of the prohibitin family of proteins that define lipid-raft-like domains of the ER. J Cell Sci 119: 3149-3160, 2006

15. Li N, Huang X, Zhao Z, Chen G, Zhang W and Cao X: Identification and characterization of a novel gene KE04 differentially expressed by activated human dendritic cells. Biochem Biophys Res Commun 279: 487-493, 2000.
16. Hu WH, Pendergast JS, Mo XM, et al: NIBP, a novel NIK and IKK(beta)-binding protein that enhances NF-(kappa)B activation. J Biol Chem 12: 29233-29241, 2005.

17. Moniz S, Veríssimo F, Matos P, et al: Protein kinase WNK2 inhibits cell proliferation by negatively modulating the activation of MEK1/ERK1/2. Oncogene 26: 6071-6081, 2007.

18. Vidal R, Calero M, Révész T, Plant G, Ghiso J and Frangione B: Sequence, genomic structure and tissue expression of Human BRI3, a member of the BRI gene family. Gene 266: 95-102, 2001.

19. Wickham L, Benjannet S, Marcinkiewicz E, Chretien M and Seidah NG: Beta-amyloid protein converting enzyme 1 and brain-specific type II membrane protein BRI3: binding partners processed by furin. J Neurochem 92: 93-102, 2005.

20. Yang Q, Inoki K, Ikenoue T and Guan KL: Identification of $\mathrm{Sin} 1$ as an essential TORC2 component required for complex formation and kinase activity. Genes Dev 20: 2820-2832, 2006. 